\title{
Another look at general covariance and the equivalence of reference frames
}

\author{
Dennis Dieks \\ Institute for the History and Foundations of Science \\ Utrecht University, P.O.Box 80.000 \\ 3508 TA Utrecht, The Netherlands
}

\begin{abstract}
In his general theory of relativity (GR) Einstein sought to generalize the specialrelativistic equivalence of inertial frames to a principle according to which all frames of reference are equivalent. He claimed to have achieved this aim through the general covariance of the equations of GR. There is broad consensus among philosophers of relativity that Einstein was mistaken in this. That equations can be made to look the same in different frames certainly does not imply in general that such frames are physically equivalent. We shall argue, however, that Einstein's position is tenable. The equivalence of arbitrary frames in GR should not be equated with relativity of arbitrary motion, though. There certainly are observable differences between reference frames in GR (differences in the way particles move and fields evolve). The core of our defense of Einstein's position will be to argue that such differences should be seen as fact-like rather than law-like in GR. By contrast, in classical mechanics and in special relativity (SR) the differences between inertial systems and accelerated systems have a law-like status. The fact-like character of the differences between frames in GR justifies regarding them as equivalent in the same sense as inertial frames in SR.
\end{abstract}

Key words: General covariance; equivalence; relativity; reference frames

Email address: dieks@phys.uu.nl (Dennis Dieks). 


\section{The meaning of equivalence}

In a striking passage of the Second Day of the Dialogue Concerning the Two Chief World Systems, Galileo (1953, p. 187) lets Salviati describe what happens in the cabin of a moving ship:

"The fish in their water will swim toward the front of their bowl with no more effort than toward the back ... the butterflies and flies will continue their flights indifferently toward every side ... And if smoke is made by burning some incense, it will be seen going up in the form of a little cloud, remaining still and moving no more toward one side than to the other. The cause of all these correspondences of effects is the fact that the ship's motion is common to all the things contained in it, and to the air also."

Everything happens as if the ship were not moving at all. Galileo is anticipating ${ }^{1}$ the relativity principle of classical mechanics here: mechanical processes take place in the same way in all inertial frames of reference. ${ }^{2}$ This is to be contrasted with what happens in accelerated frames. Because of the inertial forces that we experience in such frames we can distinguish them immediately from uniformly moving frames. Accordingly, it is duely stressed by Galileo that the water on which the ship is sailing should be calm, and the ship's motion tranquil.

The equivalence of inertial frames in classical mechanics was extended to nonmechanical processes by Einstein and became one of the two basic postulates of special relativity theory (SR). In his general relativity theory (GR) Einstein set out to generalize this principle to arbitrary frames of reference. There seems to be a consensus among philosophers of physics that he failed - a verdict that we shall try to reverse in this paper. But let us have a closer look at Galileo's discussion of the equivalence of frames before considering these modern developments.

Suppose we open the windows of Galileo's cabin: the air in the cabin will in general no longer move along with the ship (as noticed by Salvati a little further on in the Dialogue). We shall feel a breeze, whose force will depend on the ship's speed (on a windless day). Smoke will not rise vertically to the ceiling anymore, but will go with the wind. So we now can tell that the ship is moving. What happened to the equivalence of frames when we opened the windows?

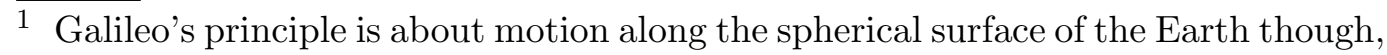
unlike the Cartesian/Newtonian principle incorporated into classical mechanics.

2 The exact sense in which this 'sameness' has to be understood will be the subject of extensive discussion below. 
The accepted reply, within the framework of classical mechanics, is that the principle according to which all inertial frames are equivalent faces no problem at all in the new situation. This is because the principle only states that the mechanical laws are the same in all inertial frames of reference; factual states of affairs may and will be different. I myself, for example, am in rest only in my own rest frame. That does not imply any conflict with the relativity principle. Similarly, the air may be at rest in the rest frame of the shores of Galileo's lake, and not at rest with respect to the ship, without repercussions for the equivalence of these frames. What counts is only whether the same mechanical laws apply in the two frames. And they do: Both in the ship frame and the lake frame, smoke is carried along by the motion of the air and butterflies fly in the air surrounding them according to the same mechanical principles.

Imagine that two crew members play a game of table-tennis in the ship's cabin. Depending on whether the windows are open or not, they will have to adjust their playing tactics; especially so if the wind is changeable. Obviously, the situations with and without wind are observationally distinguishable. But this does not entail that the frames are inequivalent in the two cases. It is not the contingent circumstance whether or not the air is moving that matters, but rather whether the motion of the ball, the motion of the air, and all other processes can be subsumed under the same general laws. The latter is the case. The differences in wind velocity correspond to different values of the parameters that specify the initial and boundary conditions, but not to differences in the applicable laws of nature.

So, our first conclusion is that Equivalence of Frames should be understood as equivalence with regard to general physical laws, not as identity of actually occurring motions or as sameness of boundary conditions. In the following we shall first elaborate on this notion of equivalence and illustrate its application to pre-relativistic physics and special relativity. We shall then come to the pièce de resistance of our analysis: the question of whether all frames of reference can be considered equivalent in the general theory of relativity.

\section{De facto or de jure}

Let us leap from classical mechanics to the early days of special relativity. From a present-day perspective ${ }^{3}$ relativity's rival was Lorentz's ether theory, according to which light moves with the constant (i.e., independent of the velocity of the source) velocity $c$ relative to the ether. Let us suppose, as is usually done, that the ether is at rest relative to Newtonian absolute

$\overline{3}$ In the first years following 1905 the physics community did not perceive Einstein's and Lorentz's theories as clear-cut rivals; see, e.g., Janssen (2002). 
space. According to the ether theory there are differences between the frame that is stationary with respect to the ether and other inertial frames: in nonstationary frames there is an 'ether wind' that one should expect to influence physical processes. Notoriously, experiments designed to measure this ether wind failed. This was part of the background of the genesis of special relativity: the null results suggested the full equivalence of all inertial frames, also with regard to optical processes - which was taken as a postulate by Einstein in 1905.

The main message of this standard quasi-historical account ${ }^{4}$ is that in the ether theory inertial frames were not equivalent, and that this was remedied by special relativity. But in the light of the discussion in the preceding section we might query this diagnosis and even doubt its intelligibility. That the ether is at rest in one frame and moving in others seems prima facie very similar to the difference between the calm in Galileo's lake frame and the breeze felt on the ship. We have placed the latter difference into the category of boundary conditions, and concluded that there is no reason to doubt the equivalence of frames because of such factual differences. Is the situation not exactly the same in the case of the ether theory? That is, is it not true that in some frames there is an ether wind, as a matter of fact, and in another frame there is no such wind, without consequence for the equivalence of these frames?

Indeed, the principles of the ether theory can be given a form that is apparently frame-independent in the same way as the principles behind the upward motion of smoke or insect flight. For example, the ether theory's light principle can be put in the form that the velocity of light is c relative to the ether - this itself is an invariant statement, valid in all frames of reference. More generally, take the Maxwell equations in their standard form, i.e., the form they take in the ether frame. Now transform them, by writing them in terms of the coordinates of an inertial frame that moves with velocity $\vec{v}$ relative to the ether. The resulting equations, in which the relative velocity of the frame with respect to the ether occurs explicitly, have a form that can be used in any inertial frame (if we now treat $\vec{v}$ not as a constant but as a quantity that is different in different frames). It remains true, of course, that the ether frame distinguishes itself from other frames because in that frame $\vec{v}=0$. But that seems analogous to the case of Galileo's lake frame, in which the air velocity vanished. Therefore, the ' $\vec{v}$-Maxwell equations' together with the laws of Newtonian mechanics appear to provide us with a formulation of the ether theory that satisfies the principle of relativity: the laws are the same in every inertial

$\overline{4}$ As is now well-known, the real history of the genesis of special relativity, the role of the null-experiments, etc., is much richer and more complicated than suggested by this 'physics textbooks account'. We are here interested, however, in the conceptual question of the meaning of 'equivalence of frames' rather than in these historical issues. 
frame of reference, only the de facto conditions vary.

As already mentioned, at the end of the 19th century it turned out that effects of motion with respect to the ether could not be measured; differences in values of $\vec{v}$ proved to be undetectable in optical and electromagnetic experiments. Lorentz attempted to find an explanation of this, within his ether theory, by postulating that all forces holding physical systems together change in the same way as electromagnetic forces when a velocity $\vec{v}$ with respect to the ether is imparted to these systems. He eventually thus managed to account for the contraction of moving measuring rods and the slowing down of moving clocks. These effects compensated other $\vec{v}$-dependent effects in the following way: the laws keep their ' $\vec{v}=0$ form' when written down in terms of 'effective', measured coordinates (coordinates determined by means of moving rods and clocks). In Einstein's decisive 1905 paper this equivalence of all inertial frames, also for optical phenomena, was transformed into an equivalence of principle, and the difference between effective and real coordinates disappeared.

But can we not say on the basis of the above considerations that the theory of the stationary ether already obeys a full principle of equivalence of all inertial frames, for mechanical as well as electromagnetic processes, even before considering Lorentz's or Einstein's innovations?

This analysis hinges on the possibility of treating the ether in the same way as the air in Galileo's example: we considered the motion of both as fact-like rather than law-like. It is clear, however, that our tentative verdict that all inertial frames are fully equivalent in the ether theory, even before Lorentz and Einstein, is completely at variance with the usual point of view. In the contemporary discussions around 1900, and also in later foundational investigations, the appearance of a value $\vec{v} \neq 0$ in the equations was always taken to signal a lack of equivalence to the 'fundamental' frame (the one at rest with respect to the ether). This suggests the presence of good reasons for doubting the analogy between the Lorentzian ether and the air surrounding Galileo's boat.

Physics texts do not even mention the possibility of regarding $\vec{v} \neq 0$ frames as equivalent in the way just explained. Although there is therefore no explicit discussion of this point, the intuition behind the judgements made in these and similar cases seems clear enough. The difference between the status of the air and that of the ether is that it is completely contingent whether the air is stationary or not in any given frame of reference: such a fact is irrelevant for the inertiality of the frame and its equivalence to other inertial frames. Physical theory does not prescribe the wind velocity: this velocity is a matter of initial and boundary conditions. On the other hand, the state of motion of the ether cannot be different from what it actually is, according to the theory of the stationary ether. In all possible universes that are described by the 
equations of the ether theory, the ether possesses the same state of absolute rest $^{5}$ and makes itself felt in exactly the same way in any chosen frame of reference. There is therefore nothing contingent about the ether wind. The state of motion of the ether cannot be different from what it actually is. It is consequently law-like rather than fact-like. That in all possible situations allowed by the theory the global space-time structure is the same, with the ether present in the same way, allows an identification of frames of reference, in the same state of motion, over different models of the theory. In all frames that rest with respect to the ether, the form of the laws with $\vec{v}=0$ applies. The difference in laws between the frames with $\vec{v}=0$ and those with $\vec{v} \neq 0$ is therefore a nomic feature of the theory rather than a mere difference in boundary conditions. ${ }^{6}$ This spoils the equivalence of these frames.

The idea behind these considerations is general and not restricted to the ether theory. For example, in the original version of Newtonian mechanics, in which the notion of rest with respect to absolute space makes sense and is even central (it occurs in Newton's first law), not all inertial frames have the same status. One inertial frame is genuinely at rest, whereas the others are in uniform motion. These different frames, with their different absolute velocities, are there in precisely the same way in all possible situations covered by Newtonian theory. This can be used to identify frames in these different situations (the velocities provide a criterion of identity of frames across different "possible worlds' - different models or solutions - allowed by the theory). Put differently, exactly the same frames of reference ${ }^{7}$, characterized by their differing states

5 Defined as rest with respect to absolute space. But one may also view the ether as something that is not placed in absolute space, but rather replaces it; as Einstein (1920) phrased it in an early historical survey of his work on relativity “... der ruhende Äther, welcher gewissermassen eine Verkörperung des von Newton eingeführten absoluten Raumes war" (the resting ether, which in a sense was a materialization of the absolute space introduced by Newton). Lorentz (1895, p. 4) himself states that it makes no sense to speak of a state of 'absolute rest' of the ether-'rest' according to him here only means that there is no relative motion between the parts of the ether. Lorentz goes on to say that all motions of celestial bodies should be regarded as defined with respect to the ether, so that it becomes clear that he has the same picture in mind as Einstein. If the ether fully takes over the role of absolute space and replaces it, it indeed becomes moot whether one can say that the ether is at rest - one can likewise question the meaningfulness of the notion of rest if applied to absolute space itself. For the subject under discussion here - the equivalence of reference frames - this question is not important. Even if absolute space is abolished, the ether remains the fixed standard with respect to which absolute velocities are defined.

6 We use the defining characteristic that laws are the same in all models of a theory, whereas facts vary from one model to another.

7 The notion of 'frame' is here meant in an abstract sense, as a congruence of worldlines in space-time, which do not have to be materially realized by moving 
of motion, are present in all models of the theory. This is not a matter of contingency but reflects a law-like feature of the theory. However, the case of Newtonian theory is atypical in that these differences do not correspond to different values of parameters occurring in the physical laws, as they did in the ether theory ( $\vec{v}$ does not appear in the equations, unlike the case of the ether theory). Newton's law-like differences between inertial frames are undetectable. This raises the suspicion that the differences in question are not needed for the formulation of an adequate dynamics. This suspicion is borne out: classical mechanics can be formulated without making use of the Newtonian notion of absolute rest. For the purpose of mechanics it is sufficient to distinguish between inertial and accelerated motion, and this can be done within a weaker space-time framework than that of Newton's theory: we do not need a notion of identity of spatial points over time. The resulting weaker structure is known as neo-Newtonian spacetime (e.g., Earman, 1989, Chapter 2.4). Against this new spatio-temporal background different states of uniform motion can no longer be used to identify inertial frames across models of the theory. It remains true, of course, that in any model of the theory inertial frames of reference move uniformly with respect to each other, but there is no way to use these uniform velocities to identify frames in different models of the theory. Remember that such an identification across models could be achieved in the original Newtonian version of the theory through the different relations of the various frames to the constant space-time background, which is present in all possible situations governed by the theory. Since velocity with respect to neo-Newtonian space is not definable, the new version of the theory no longer has the conceptual resources to establish this rigid reference connection between inertial frames in different models.

Consequently, in neo-Newtonian spacetime there are no longer law-like differences between inertial frames. They all become fully equivalent. But acceleration still is a well-defined property of frames of reference, since neo-Newtonian spacetime is equipped with a notion of four-dimensional straightness. What was true for absolute velocities in the original theory now applies to accelerations (i.e., lack of straightness of worldlines). There is a law-like difference between accelerated and non-accelerated frames; and this difference cannot be dealt with as something that is merely contingent.

To be more accurate we should point out that the identification across models is not always in terms of individual frames, but may pertain to classes of frames possessing the same defining characteristics. Thus, in the neo-Newtonian case the value of the acceleration only picks out a class of accelerated frames, which are in uniform motion with respect to each other.

In summary, both in the case of the ether theory and that of Newtonian

$\overline{\text { systems. }}$ 
classical mechanics there are nomological, law-like, differences between frames. A natural way of capturing these differences is to say that the different frames are present in the same way in all solutions allowed by the equations of the theory; that all models of the theory contain them in the same way. To take the ether as an example: the ether has the same properties in all possible worlds (as defined by the theory) and serves as a fixed skeleton with respect to which frames can be defined. This provides a natural criterion of identity of frames across models.

\section{Law-like features, rigid reference and absolute objects}

The a priori fixed character of the ether and Newtonian space-time structure can alternatively be expressed by saying that these constitute 'absolute objects', in the sense of Anderson (1967, pp. 83-87). Anderson defined absolute objects as spatio-temporal features that affect physical processes, but are not themselves affected by anything. He used these 'objects' to define the symmetry group of the theory: this is the group of space-time transformations that leave the theory's absolute objects invariant. In Lorentz's theory the ether is absolute and the symmetry group is the group of translations and rotations, since these leave the ether the same (there are no preferred points or directions in the ether). The fundamental frame, i.e., the frame at rest with respect to the ether, is invariant under the transformations of this symmetry group. More generally, frames from one equivalence class of frames are transformed into each other by elements of the theory's symmetry group.

The result of Anderson's analysis with regard to the question of which frames are equivalent in classical mechanics and special relativity, and which transformations connect these frames, is the same as what we argued for in connection with law-like equivalence above. However, Anderson introduced his absolute objects by contrasting them with 'dynamic objects', which he defined as entities whose state parameters occur in a variational principle associated with the equations of motion. From a conceptual point of view it is unclear, however, why such a connection with the form of the dynamics should be central to the analysis of physical equivalence of frames of reference. Indeed, it may very well be and must even be expected that objects like the structure of neo-Newtonian space-time and the $g_{\mu \nu}$ of special relativity can be given a dynamical status: all one has to do is to formulate variational principles that invariably have these structures as their solutions (Maidens, 1998). By focusing on sameness across models and linking this to the distinction between law-like and fact-like features of reference systems, as we are doing here, the relevance of 'absoluteness' for the equivalence of frames does become perspicuous. Since the absolute objects occur in each and every model of the theory, they can serve to connect frames that occur in different models: (classes of) frames that possess the same 
relations to the absolute structure can be identified.

The absolute objects thus serve as the fixed background with respect to which identity across models is defined. The fixed background itself occurs in the same way in all models of the theory. One may wonder how this 'sameness' should be characterized mathematically, since the precise form of a technical criterion for the identity of geometric objects across models is a debated subject in the literature (Friedman, 1983; Torretti, 1984; Maidens, 1998). For our purposes here we do not need to give a definitive general answer to this question, though. It is enough for us to specify what the absolute objects are in the theories we are considering: (neo-)Newtonian space-time structure in classical mechanics and the Minkowski metric in special relativity. These space-time structures are the nomic backbones in all models of these theories. There is no similar invariable space-time structure in general relativity.

The problem of how to formulate completely general identity criteria across models that can pick out fixed structure in all imaginable space-time theories need therefore not immediately concern us. For our purposes and the theories we are discussing the criterion of diffeomorphic equivalence proposed by Friedman (1983) seems satisfactory. In the 'coordinate language' that was common until coordinate-free formulations of space-time theories became dominant, a couple of decades ago, two models are diffeomorphically equivalent if a coordinate transformation in one model is possible that makes its coordinate description identical to that of the other model. Space-time structure is absolute if it can be given the exact same appearance in all models of the theory through suitable choices of coordinates. For example, the Minkowski inertial structure is absolute in special relativity because a choice of coordinates is possible in each model in terms of which $g_{\mu \nu}=(-1,1,1,1)$ everywhere.

It has been questioned whether this criterion of diffeomorphic equivalence adequately captures the notion of absolute structure in general. For example, Torretti (1984, p. 285) argues that an imaginary theory that would postulate that space-time has a certain constant and non-dynamical curvature, but remains silent about the concrete value of this curvature, possesses models with different curvatures. Such models are not diffeomeorphically equivalent (because curvature is invariant under diffeomorphisms). The curvature would therefore not qualify as an absolute object here, in conflict with intuition. However, this consequence disappears as soon as we reformulate this theory in a way that brings it closer to actually existing theories. The case described by Torretti would ordinarily be regarded as a case in which we are ignorant about the exact form of the theory we should use: there are different possibilities, each with its own class of models (characterized by one value of the curvature). In each one of these theories curvature does qualify as an absolute object - on the basis of the criterion of diffeomorphic equivalence - so that the conclusion would become that curvature is absolute, whatever the form of the 
right theory is. On another interpretation of Torretti's example (not meant by its author) the theory would describe a collection of possible universes, each with its own curvature. In this case curvature would fail to qualify as absolute but this would not seem to conflict with intuition, because curvature really is a non-constant feature of the theory. Another objection, which is more directly relevant from this paper's viewpoint because it concerns GR, is the one put forward by Geroch - as reported by Friedman (1983, p. 59). It relies on the fact that any two everywhere non-vanishing time-like vector fields are diffeomorphically equivalent (because their integral curves can be mapped onto each other by a diffeomorphism). So in cosmological models in which there is omnipresent dust, the associated velocity fields would qualify as absolute objects. However, this conclusion does not have repercussions for GR as such: the set of all models of GR certainly contains models in which there is no dust in finite space-time regions, so the diffeomorphism criterion deems dust velocity fields to be not absolute in the actual GR.

To restrict oneself to a smaller class of models, in all of which the universe is completely full of dust, means changing the theory. It is true that the new theory that comes into being when only such dust-filled universes are admitted does treat the dust velocity field as absolute. This does not conflict with expectations: in all worlds allowed by this new theory there is a congruence of dust worldlines that can serve as a background to fix time-like coordinate axes. There would be an absolute frame in this imaginary theory: the restframe of the dust. It would consequently be possible to systematically identify (classes of) other frames through their states of motion with respect to this dust frame. It is thinkable that the theory could be such that there is an associated systematic variation in the parameters occurring in the laws as they apply to these frames (e.g., this could be due to interaction with the dust, assuming it is the same kind of dust that occurs in all models). In this case there would be nomic differences between frames - as far as I can see this is not in conflict with what intuition tells us about such dust worlds. It would be interesting to see whether there are other cases in which the criterion of diffeomorphic equivalence does give rise to unacceptable results about the equivalence of frames.

In summary, fixed or 'absolute' structure yields the possibility of identifying features across models of the theory. It thus becomes part of the theory's nomic structure. Differences between frames that are due to different relations these frames bear to the fixed backdrop are to be considered law-like rather than fact-like. In particular, if the laws of motion in these frames contain quantities that characterize the different absolute motions of these frames (like position, velocity or acceleration with respect to the fixed background), these laws are to be regarded as different. By contrast, if only contingent boundary or initial conditions vary, the frames in question are equivalent. This analysis of equivalence of frames is in accordance with the way these notions are usually 
employed both in pre-relativistic physics and in special relativity.

\section{Special relativity}

In special relativity there is no ether or absolute space. Still, there is a fixed spatio-temporal geometric structure, similar to that of neo-Newtonian spacetime, namely Minkowski space-time. Possible worlds according to special relativity are specifications of particle and field configurations within identical copies of Minkowski space-time. Inertial frames are defined by congruences of time-like geodesics in this absolute space-time structure. In coordinates adapted to these frames we have $g_{\mu \nu}=(-1,1,1,1)$.

We could now follow the recipe of sec. 2 and subject these laws to arbitrary coordinate transformations. In this way we can obtain a generalized form of the laws, valid in coordinates adapted to any frame of reference. This maneuver

was famously proposed by Kretschmann (1917, p. 579). Kretschmann started from the standard form of the special relativistic law of light propagation, $\triangle x^{2}-\triangle t^{2}=0$. Writing this in 'generally covariant form' he obtained

$$
\begin{aligned}
\delta \int d s & =0 \\
d s^{2} & =0 \\
R_{\mu \nu \tau}^{\lambda} & =0 .
\end{aligned}
$$

The last of these equations states that the Riemann tensor vanishes identically.

The same thing can be done for inertial particle motion, which leads to

$$
\begin{aligned}
\delta \int d s & =0 \\
R_{\mu \nu \tau}^{\lambda} & =0 .
\end{aligned}
$$

The vanishing of the Riemann tensor ensures the flatness of space-time (a stipulation about the topology should be added to make sure that we are in global Minkowski space-time). Although the sets of eqs. (1) and (2) look the same in any coordinate system, they are equivalent with the original equations. And obviously, the differences between inertial and accelerated frames cannot change as a result of this mere rewriting procedure.

The way in which these differences are represented in the equations becomes clear when we work out eqs. (1) and (2). For the case of particle motion we 
obtain

$$
\frac{d^{2} x^{i}}{d s^{2}}=\Gamma_{j k}^{i} \frac{d x^{j}}{d s} \frac{d x^{k}}{d s}
$$

where $\Gamma_{j k}^{i} \equiv g^{i l}\left(g_{l j, k}+g_{l k, j}-g_{j k, l}\right)$ are the so-called Christoffel symbols (with $g_{i j}$ the coefficients of the line element $d s^{2}=g_{i j} d x^{i} d x^{j}$ in arbitary coordinates and commas denoting differentiation). Eq. (3) generalizes the inertial motion equation $d^{2} x^{i} / d t^{2}=0$. It applies both to inertial and accelerated systems. But despite this form invariance there is no equivalence of frames. Indeed, by virtue of the last formula in both Equation (1) and (2) (plus a stipulation about the topology) the space-time always contains a set of global inertial frames and incorporates a notion of absolute acceleration. The numerical values of the coefficients $\Gamma_{j k}^{i}$ that occur in the 'generally valid form' of the equation of motion have therefore the same status as the $\vec{v}$ of sec. 2: they are quantities that encode the acceleration of the frame that is used - the (possibly variable) deviation of straightness of the worldlines of the congruence that defines the frame. These numbers identify the class of equivalent frames to which our frame belongs, in a way that is the same in all models of the theory. Applying the ideas of the preceding sections, we conclude that the mere sameness of form of the eqs. (3) in all frames of reference does not entail the equivalence of these frames. Accelerated frames are nomically different from inertial frames: the differences are codified in the values of the $\Gamma_{j k}^{i}$.

\section{Gravitation}

The symmetry between inertial frames and non-inertial frames in special relativity is thus broken by the law-like status of the difference between 'inertiality' and 'non-inertiality'. It is true that the equations can be written in such a way that they have the same appearance in every frame of reference, but this does nothing to remove the rigid reference that is made, in these same equations, to the class of frames in which they apply. There is therefore no general equivalence of frames in special relativity. Rewriting the theory in generally-covariant form does nothing to change this.

Consequently, in order to achieve the equivalence of all frames we should see to it that there no longer is a fixed background with respect to which classes of frames can be identified across different models of the theory. The characteristics of the motions as described within a frame, or the values assumed by $\Gamma_{j k}^{i}$, should not be usable as address labels with the help of which classes of frames can be located with respect to a fixed structure that is the same in all models of the theory. (As we have seen, in special relativity this is possible, because the $\Gamma_{j k}^{i}$ - once we know in which way the coordinates have been 
assigned - contain information about the linear acceleration and rotation of the considered frame.)

The way to accomplish this is to make sure that the structure that is fixed in all solutions of the theory is weakened, so that the values of $\Gamma_{j k}^{i}$ can no longer function as address labels. The transition from Newtonian space-time to neoNewtonian space-time affords an example of such a weakening of space-time structure. In Newtonian space-time different velocities relative to absolute space distinguish between inertial frames, but in neo-Newtonian space-time it is no longer possible to define such different velocities. This results in the full equivalence of all inertial systems. If we could make a similar move in relativity theory, by weakening Minkowski structure in such a way that acceleration is no longer definable on the basis of the fixed background structure, this would solve our problem.

However, there is a decisive point on which the situation in special relativity deviates from that in Newtonian mechanics: absolute acceleration in special relativity is an observable quantity (as it is in classical mechanics), whereas absolute velocity in Newtonian theory is notoriously unobservable. Because of the latter fact, the transition from Newtonian to neo-Newtonian space-time is possible without observational consequences. Such an effortless change is not in the cards in the case of relativity theory. The requirement that inertial forces do not provide a rigid reference to classes of frames must involve going over to a theory that treats these forces as contingent, i.e., depending on initial and boundary conditions.

It is here that Einstein's principle of equivalence enters. This principle offers prospects of replacing absolute acceleration with a quantity that is comparable to gravitational acceleration in Newton's theory, conditioned by contingent factors like the distribution of masses. We can thus hope to treat the phenomena that testify to the non-inertial character of frames - e.g., the occurrence of Coriolis and centrifugal forces - as contingently occurring phenomena that do not count against the equivalence of these frames with inertial ones. The occurrence of inertial forces could be like the appearance of a breeze in Galileo's cabin with open windows; or like the appearance of magnetic forces in frames that move with respect to electrical charges. To elaborate on the latter example: a special relativistic rest frame in which there are only Coulomb forces might be said to be privileged in a de facto sense. But since the same Maxwell equations hold in all inertial frames in special relativity, without any differences in the numerical values of the constants occurring in them, the differences between the electrical forces in the various frames are completely due to differences in initial and boundary conditions. Accordingly, in special relativistic electrodynamics such differences are never interpreted as signifying fundamental distinctions in the status of frames of reference. 
That a similar account of inertiality is possible is suggested by Einstein's equivalence principle. This principle states that being in a frame that accelerates with respect to a (special relativistic) inertial frame and being in an inertial system in which there is a homogeneous gravitational field are equivalent situations: the difference is one of description. This hints at a dynamical explanation of the difference between inertiality and non-inertiality: the distinction may be similar to the distinction between frames without and with a non-static electromagnetic field-but now gravitation instead of electricity and magnetism will have to do the job. ${ }^{8}$

Obviously, this idea can succeed only if the gravitational field is a dynamic entity, subject to a field equation. Nothing would be gained if 'gravitational field' would be just a label for referring to an a priori given space-time structure. The latter might be suggested by Einstein's original form of the equivalence principle, according to which a homogeneous gravitational field (actually, not a solution of the Newtonian gravitational equation with non-vanishing sources) is indistinguishable from a uniform acceleration field. We have to look for a dynamic theory of gravitation. Newtonian gravitational theory affords the natural starting point: it supplies us with an equation that determines a gravitational field given sources and boundary conditions (as described from a given inertial frame of reference). If this gravitational field does not vanish, this can in the light of the equivalence principle be taken to mean that we are in a frame that is accelerated, while the acceleration has the status of something contingent, depending on the distribution of material sources. If such a strategy works out, the presence of an acceleration field, or inertial forces, will no longer be a reason to think a frame is fundamentally different from a frame without such effects.

8 Janssen $(2005,64)$ points out the crucial importance, also in Einstein's actual thinking, of this analogy between the treatment of electrical and magnetic fields in special relativity on the one hand and that of inertial and gravitational fields in general relativity on the other. He concludes that with GR Einstein achieved a 'relativity of the inertial-gravitational field' but not the relativity of arbitrary motion (because frames that accelerate with respect to each other are clearly physically distinguishable; being in a rapidly spinning spacecraft feels very different from being in free fall). The analysis of the present paper dovetails very nicely with this conclusion. We add that it is this relativity of the inertial-gravitational field (itself a consequence of the form invariance of the laws of nature) and not relativity of motion that is the essential criterion for judging whether or not frames are equivalent. 


\section{General relativity}

The principle of equivalence was one of the heuristic stepping stones in the genesis of general relativity. However, the final theory does not always agree with the ideas that guided its formation. One point is that although general relativity is certainly a theory of gravitation, the notion of a gravitational force and a gravitational field do not appear in the theory - at least not in the form in which they occur in Newton's theory of gravitation. Gravitation has become geometrized: the field equations determine the metric field $g_{\mu \nu}(x)$, which in turn encodes all gravitational effects.

This does not constitute an obstacle, however, for carrying through the program sketched in sec.5. We can define coordinate systems in which $g_{\mu \nu}=$ $(-1,1,1,1)$ as being adapted to inertial systems; frames whose adapted coordinates do not lead to these $g_{\mu \nu}$ values are by definition non-inertial. This definition translates the special relativistic notion of inertial system into the framework of general relativity. It entails that in general inertial systems are only defined locally.

The situation is now the one envisioned in sec. 5. We solve the Einstein equations, given the mass-energy distribution in the universe plus boundary conditions, in terms of an arbitrary coordinate system; by coordinate transformations we then establish what the local inertial system are. Systems that accelerate with respect to these local inertial frames are non-inertial. The physical differences between the inertial and non-inertial systems are of course still what they were before: in non-inertial frames there are Coriolis, centrifugal and similar effects. But these differences have now become the result of contingent factors. The boundary and initial conditions with which the Einstein equations have to be combined decide the patchwork pattern of local inertial frames. It is not determined a priori which frames will be inertial and which ones will not. There is no fixed structure that can serve to identify frames across all models of the theory: the differences in frames are contingent rather than law-like. In Galileo's example, the rest frame of the lake (in which the air was stationary) was physically different from other frames, but that did not imply a fundamental difference in status. In the same way here, the local inertial frame is different from non-inertial frames without a violation of the fundamental law-like equivalence of these frames.

\section{Covariance, symmetry and relativity}

One of the main ideas behind calling the relativistic theory of gravitation general relativity was the idea that the new theory generalizes the relativity 
principle of the special theory. Einstein saw this generalization realized in his principle of general covariance, according to which the laws should take the same form in all coordinate systems. He claimed that this principle was satisfied in GR but not in SR, so that we have a genuine generalization here.

There is, however, a long tradition in which Einstein's view is criticized, starting with the already mentioned paper by Kretschmann (Kretschmann, 1917). Kretschmann argued that the requirement of general covariance is physically vacuous and therefore unconnected to any relativity principle. A popular modern version of this line of criticism maintains that Einstein confused covariance and space-time symmetry (Friedman, 1983). ${ }^{9}$ The argument is that the form invariance of the laws, required by the principle of general covariance, can always be achieved by choosing clever mathematical formulations of the laws. We have seen the correctness of this claim in secs. 2 and 4 . The requirement of general covariance does therefore not impose any restrictions on space-time theories. By contrast, the argument continues, what is at stake in a relativity principle is the invariance of $g_{\mu \nu}$ in the transition from one reference frame to an equivalent one. In other words, equivalent frames are connected by transformations from the space-time symmetry group. There can thus only be a non-trivial relativity principle in theories in which the space-time exhibits symmetries. But in GR the symmetry group of $\left\langle M, g_{\mu \nu}\right\rangle$ is the identity group, because there is no fixed space-time structure common to all models of the theory. This then would show that there is no general relativity and no general equivalence of frames in GR at all.

The idea behind this linking of a relativity principle to symmetries of the space-time would seem to be that the spatio-temporal background should look the same from equivalent frames. This can be justified to the extent that in this case the choice of the same initial and boundary conditions will lead to the same solutions of the equations of motion in the different frames. However, space-time itself is left out of consideration in the specification of the boundary conditions, which shows that this conception of 'relativity' is tailored to theories in which space-time structure is a priori rather than subject to dynamical laws. The analysis proposed in this paper extends this notion of relativity to the more general case in which space-time itself may be dynamical. We have therefore insisted on the difference between laws and contingent, factual, conditions in a quite general way, which includes the case of laws governing the space-time structure. These general laws should be the same in equivalent frames. As we explained, this entails that the laws in different but equivalent frames should not contain different references to a fixed background structure. In the case of special relativity this leads to the conclusion that only frames connected by Lorentz transformations are equivalent. But in GR the differences between all frames can be said to be purely factual.

$\overline{9}$ See (Norton, 1995) for a review of the discussion. 
So Kretschmann was right in that laws can always be given the same form, in the syntactic sense of being given by the exact same string of symbols, and that this does not guarantee the equivalence of frames. The essential question, however, is not about this syntactic form invariance, but about whether there are law-like differences between frames. As illustrated by the equations involving $\vec{v}$ in sec. 2, we can have expressions that have the same syntactical form and yet do not represent the same law because of semantic considerations. If we interpret the principle of general covariance as the requirement that the same laws hold in all coordinate systems, i.e. without quantities entering into the expressions that locate the system with respect to a fixed space-time background, there $i s$ a relation with the equivalence of frames. In a general principle of relativity we have to restrict the class of admissible laws to those not containing reference to a fixed space-time background. This is very close to the suggestions made by Weyl (1918, secs. 26, 27) and Pauli (1921, sec. 52). The difference is in the conceptual justification: Weyl and Pauli start by imposing the general requirement that laws should only contain "physical state quantities, to which something real corresponds" (physikalische Zustandsgrößen, denen etwas Reales entspricht), and in this way attempt to block the introduction of mathematical devices that 'artificially' give the laws the same form in every frame. By this strategy they achieve that in Newtonian mechanics and special relativity not all frames become equivalent, in spite of the possibility of a covariant formulation of the laws. For example, in special relativity the quantities $\Gamma_{j k}^{i}$ (see sec. 4 ) occur in the generally covariant equations; but these quantities are not subject to a dynamical law and therefore not physically meaningful, according to Weyl and Pauli. By contrast, in general relativity the $\Gamma_{j k}^{i}$ are determined by the dynamical Einstein equations.

The problem with this proposal, however, is that the connection between 'physically real' and 'non-dynamical' is completely unclear. Why should the $\Gamma_{j k}^{i}$ from sec. 4 not be physically significant (compare Norton, 1995, pp. 232233)? The space-time structures of classical mechanics and special relativity are certainly meant to correspond to things that are physically very real and significant. That the laws should not contain non-dynamic quantities of the type we have discussed can therefore not be derived from the requirement that only physically significant or real quantities should occur in them.

In terms of its final conclusions the analysis defended here comes close to proposals made earlier in the literature. Only the connection with the distinction between law-like and fact-like features, however, makes the idea cogent from a conceptual point of view. General covariance in the sense given here is satisfied in GR, and guarantees the equivalence of all frames of reference in this theory. 


\section{Einstein}

The conclusion of the preceding sections suggests that Einstein was right after all: that general covariance does imply general equivalence of frames and that GR is a direct generalization of SR. This verdict depends, evidently, on the interpretation given here to the notions of general covariance and equivalence of frames. Can this analysis be seen as a fair gloss of Einstein's own pronouncements on these issues and thereby furnish a justification of his position?

A straightforward answer to this question is made difficult by the fact that there are several intertwined strands discernible in Einstein's early thinking about general covariance and relativity. I do think that essential elements of the approach defended here constituted one of these strands. But there were also considerations relating to a general empiricist stance in the philosophy of science, to Mach's principle and the relational character of motion à la Leibniz that played a role, especially in the early period of Einstein's work on general relativity (1907-1918). ${ }^{10}$ I believe, however, that ideas in the spirit of what we have discussed here remained important for Einstein during his whole life, whereas the themes of empiricism, Mach's principle and the relativity of motion gradually faded away.

In sec. 5 we already commented on the part played by the analogy between electromagnetism and gravitation, via the principle of equivalence. As Einstein (1920, p. 265) stressed in his early historical comments on the genesis of general relativity, this analogy alerted him to the possibility of seeing the occurrence of inertial effects as something of the same status as the occurrence of an electric field in a frame in which a magnet moves. He concludes in this document that there is no essential difference between an accelerating and an inertial frame - the differences are fact-like, in our terminology-so that all frames can be considered as equivalent. In his review article of the just-finished theory, Einstein (1916) introduced his subject by pointing out a serious defect in special relativity: this theory defines the difference between inertial and non-inertial frames through their different relations to absolute space. He then stressed that the only satisfactory approach would be one in which the differences in question would be related to differing situations of the frames with respect to physical things, such as distant masses, while the general laws of motion would remain the same in all frames. This clearly is in the spirit of the idea elaborated here (although we would allow boundary conditions for the metric field alongside distant matter). Einstein kept stressing this relation between equivalence and sameness of the laws during his whole career (Norton, 1995, p. 238). In his Autobiographical Notes (Schilp, 1949, p. 66) Einstein wrote with approving hindsight about his thoughts in

\footnotetext{
${ }^{10}$ Cf. Janssen (2005) for the confusing role played by Mach's principle.
} 
1908 (with slight changes in Schilp's translation in order to make the rendition more literal):

"So, if one regards as possible gravitational fields of arbitrary extension, not a priori restricted by spatial boundary conditions, then the concept 'inertial system' becomes completely empty. The concept 'acceleration with respect to space' then loses all meaning and with it the principle of inertia together with Mach's paradox. Thus, the fact of the equality of inert and heavy mass leads quite naturally to the conception that the basic requirement of the special theory of relativity (invariance of the laws under Lorentz transformations) is too narrow, i.e., that one has to postulate invariance of the laws also with respect to non-linear transformations of the coordinates in the four-dimensional continuum." 11

The ideas of rejection of absolute space as a fixed structure, its replacement by a dynamical mechanism (gravitation) and the relation of this to form invariance of the laws are all clearly present here. In the second appendix of the last edition of 'The Meaning of Relativity' (Einstein, 1956, p. 139-140) we find something very similar:

"It is the essential achievement of the general theory of relativity that it has freed physics from the necessity of introducing the 'inertial system' (or inertial systems). This concept is unsatisfactory for the following reason: without any deeper foundation it singles out certain coordinate systems among all conceivable ones. It is then assumed that the laws of physics hold only for such inertial systems (e.g. the law of inertia and the law of the constancy of the velocity of light). Thereby, space as such is assigned a role in the system of physics that distinguishes it from all other elements of physical description. It plays a role in all processes, without in its turn being influenced by them."

Einstein (1956, p. 141) then goes on to discuss the improvement introduced by general relativity, by comparing it with Gauss's theory of curved surfaces:

"Since, in general, there exists no preferred coordinate system on a surface,

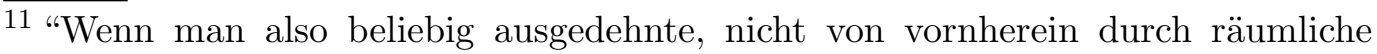
Grenzbedingungen eingeschränkte, Gravitationsfelder als möglich betrachtet, so wird der Begriff des Inertialsystems völlig leer. Der Begriff 'Beschleunigung gegenüber dem Raume' verliert dann jede Bedeutung und damit auch das Trägheitsprinzip samt dem Mach'schen Paradoxon. So führt die Tatsache der Gleichheit der trägen und schweren Masse ganz natürlich zu den Auffassungen, dass die Grund-Forderungen der speziellen Relativitätstheorie (Invarianz der Gesetze bezüglich Lorentz-Transformationen) zu eng sei, d.h. dass man eine Invarianz der Gesetze auch bezüglich nicht linearer Transformationen der Koordinaten im vierdimensionalen Kontinuum zu postulieren habe." 
this investigation led for the first time to expressing the relevant quantities in general coordinates. Riemann has extended this two-dimensional theory of surfaces to spaces of an arbitrary number of dimensions ..."

In any particular surface there clearly may be preferred coordinates, adapted to the spatial structure of that surface. Evidently, what Einstein wants to express is that if we consider all possible surfaces, there is no common fixed metric skeleton, to which we could adapt coordinate systems by means of a general recipe. In other words, within the set of all curved surfaces there is no a priori fixed structure that could serve to relate coordinate systems on different surfaces. All coordinate systems get therefore equal a priori rights. It is the analogous feature in four dimensions that, in the transition from special to general relativity, removes the special status of inertial systems and guarantees the general validity of the laws of physics. This is the same thought as the one underlying the proposal of this paper.

However, in his reply to Kretschmann Einstein (1918) notoriously chose a different strategy. He admitted that Kretschmann was right in his claim that general covariance was more a matter of mathematical ingenuity in writing down the equations than of physical content. Nevertheless, he emphasized the heuristic value of general covariance if combined with a principle of simplicity. So it may seem that at this crucial moment Einstein was following an analysis of general covariance that is at variance with the one proposed here. Worse, it appears that his reliance on simplicity is hardly a move that can be taken seriously: there are no general arguments that show that simplicity improves a theory's chances of being true, and the notion of simplicity itself is notoriously shaky.

On the other hand, the obviousness of such general objections to the value of the notion of simplicity makes one wonder what concrete 'simple' features Einstein had in mind. The example Einstein (1918) gives is that of Newtonian theory versus general relativity: he claims that from the point of view of the absolute differential calculus Newton's theory is forbiddingly complicated. As Norton (1995) points out, this example is unfortunate. Indeed, it would not take long before four-dimensional formulations of Newtonian theory that did not look terribly complicated appeared on the stage. On the other hand, it is plausible that Einstein's expectation that such a theory would be very troublesome was based on his conviction that the fixed space-time structure of Newtonian theory, consisting of separate spatial and temporal parts as it does, would resist the kind of elegant simple covariant formulation general relativity is capable of. In other words, it may well be that Einstein's appeal to simplicity was an expression of his intuition that a correct theory should be able to do without the complication of a fixed space-time background: less a priori structure certainly adds to the simplicity of a theory, from one point of view. This interpretation receives support from what Einstein writes 
about this issue in his Autobiographical Notes (Schilp, 1949, pp. 68,69). ${ }^{12}$ After conceding again that a mere rewriting of expressions can make them look generally covariant, he stresses the great heuristic significance of searching for equations that in their generally covariant formulation are as simple as possible. He adds that if this simplicity is achieved, fields that can be transformed into each other by the allowed general transformations will describe the same real state of affairs. The idea apparently is that in this case only mutual relations between the dynamical fields are important in the specification of the physical state of affairs. The absence in physical reality of rigid background structure that would not remain invariant under all transformations seems the basic intuition behind all these considerations.

In his reply to Kretschmann, Einstein (1918) expressed the same thought by his 'point-coincidence principle': the laws of nature merely express spatiotemporal coincidences. This principle already occurred in the 1916 review paper as an argument for general covariance. But in the 1918 paper, Einstein emphasized the need to distinguish the relativity principle from Mach's principle and the relational character of motion, stating that he had not been clear on this issue before. He now stated that the point-coincidence principle captured the exact content of the relativity principle, and claimed that this principle has the consequence that the only natural form of the laws must be generally covariant. The way I read this is that the point-coincidence principle contains the tacit assumption that it is only 'physical things' (particles and fields - and not a priori determined objects) and their coincidences that play a role in the specification of the state of the universe and in the laws of nature. The point-by-point coincidences between these dynamical objects contain everything there is to know about the world. Fixed space-time structure is assumed to be absent. ${ }^{13}$ After this paper (Einstein, 1918), Mach's principle and the relativity of motion gradually lost their significance in Einstein's theorizing. But that is a different story, with its own complications.

Arguments along these lines suggest broad agreement between Einstein's own position and what is proposed in this paper. But I do not want to press the point. It is sufficient for my purposes here if the analysis of general covariance and equivalence of frames presented here can stand on its own two feet, regardless of whether Einstein had something similar in mind.

\footnotetext{
12 Einstein's later recollections concerning his trajectory to GR should be handled with care. See, e.g., van Dongen (2002) and Janssen and Renn (forthcoming). However, as far as the notions of covariance and equivalence of frames are concerned there is a remarkable consistency in Einstein's writings, at least regarding the aspects dealt with in this paper.

${ }^{13}$ For this reason, the point-coincidence principle guarantees that the diffeomorphically related cosmological models discussed in the hole argument satisfy the same laws.
} 


\section{References}

Anderson, J. L. (1967). Principles of relativity physics. New York: Academic Press.

Earman, J. (1989). World enough and space-time. Cambridge, MA: MIT Press.

Einstein, A. (1916). Die Grundlage der allgemeinen Relativitätstheorie. Annalen der Physik, 49, 769-822.

Einstein, A. (1918). Prinzipielles zur allgemeinen Relativitätstheorie. Annalen der Physik, 55, 240-244.

Einstein, A. (1920). Grundgedanken und Methoden der Relativitätstheorie, in ihrer Entwicklung dargestellt. Doc. 31, pp. 245-282 in: Janssen, M. et al. (Eds.), The collected papers of Albert Einstein, Vol. 7. The Berlin years. Writings: 1918-1921. Princeton: Princeton University Press, 2002.

Einstein, A. (1956). The meaning of relativity. fifth edition. Princeton: Princeton University Press.

Friedman, M. (1983). Foundations of space-time theories. Princeton: Princeton University Press.

Galilei, G. (1953). Dialogue concerning the two chief world systems. Berkeley: University of California Press. Italian original: 1632.

Janssen, M. (2002). Reconsidering a scientific revolution: the case of Einstein versus Lorentz. Perspectives on Science, 10, 457-522.

Janssen, M. (2005). Of pots and holes: Einstein's bumpy road to general relativity. Annalen der Physik, 14, Supplement, 58-85.

Janssen, M., and J. Renn (forthcoming). Untying the knot. How Einstein found his way back to field equations discarded in the Zurich notebook. Pp. 347433 in: Renn, J. (Ed.), The genesis of relativity. Vol. 2. General relativity in the making: Einstein's Zurich notebook, commentary and essays. Berlin: Springer.

Kretschmann, E. (1917). Über den physikalischen Sinn der Relativitätspostulate. A. Einsteins neue und seine ursprüngliche Relativitätstheorie. Annalen der Physik, 53, 575-614.

Lorentz, H. A. (1895). Versuch einer Theorie der electrischen und optischen Erscheinungen in bewegten Körpern. Leiden: Brill.

Maidens, A. (1998). Symmetry groups, absolute objects and action principles in general relativity. Studies in History and Philosophy of Modern Physics, 29B, 245-272.

Norton, J. D. (1995). Did Einstein stumble? The debate over general covariance. Erkenntnis, 42, 223-245.

Pauli, W. (1921). Relativitätstheorie (Sonderabdruck aus der Encyklopädie der mathematischen Wissenschaften). Leipzig: Teubner. Theory of relativity. London: Pergamon, 1958.

Schilp, P. A. (Ed.) (1949). Albert Einstein: philosopher-scientist. La Salle: Open Court.

Torretti, R. (1984). Spacetime physics and the philosophy of science. British Journal for the Philosophy of Science, 35, 280-292. 
van Dongen, J. (2002). Einstein's unification: general relativity and the quest for mathematical naturalness. Ph.D. dissertation, University of Amsterdam.

Weyl, H. (1918). Raum-Zeit-Materie. Berlin: Springer. Space-time-matter. New York: Dover, 1952. 